\title{
Allylamine-Adducted Bismaleimide Resins. II. Their Physical/Mechanical Properties and Morphology
}

\author{
KING-FU LIN* and JIN-SING LIN \\ Institute of Materials Science and Engineering, National Taiwan University, Taipei, Taiwan 10764, R.O.C.
}

\begin{abstract}
SYNOPSIS
Physical/mechanical properties of various cured allylamine-adducted bismaleimides (ABMI), such as allylamine-adducted 4,4'-bismaleimidodiphenylmethane (A-BDM), and allylamine-adducted 4,4'-bismaleimidodiphenylether ( $A-B D E$ ) were investigated and correlated to their morphology. In the previous study, two types of the curing reactions were found: one is the homopolymerization through the opening of double bonds in the maleimide groups and the other is the reactions between allyl groups and benzene rings. Dynamic mechanical analysis and scanning electron microscopy of cured A-BMI indicate that the network produced by homopolymerization is immiscible with that produced by the other reactions. The glass-transition temperature $\left(T_{g}\right)$ of the former network is lower than that of the latter. In addition, the 50\% A-BDM prepared by reacting BDM with $50 \mathrm{~mol} \%$ allylamine has the optimal physical/mechanical properties after cure, such as the highest $T_{g}$ and the highest flexural strength and modulus among the A-BDM. Moreover, the decomposition temperature of $50 \% \mathrm{~A}-\mathrm{BDM}$ is $450^{\circ} \mathrm{C}$, only about $40^{\circ} \mathrm{C}$ lower than that of the pure BDM. (C) 1994 John Wiley \& Sons, Inc.
\end{abstract}

\section{INTRODUCTION}

The cured bismaleimides (BMI) are extremely brittle because of the highly cross-linked structure. To improve their toughness and processability, the cross-linking density of BMI was reduced by using the diamine as a chain extender through the Michael addition. ${ }^{1-5}$ However, the chain extension usually induced the side effects on the properties of cured BMI, such as poor thermal stability. ${ }^{1}$ The other excellent approach ${ }^{6}$ was to use the $o, o^{\prime}$-diallylbisphenol $\mathrm{A}$ as a toughening agent for $4,4^{\prime}$-bismaleimidodiphenylmethane (BDM) resin, which not only improves the toughness and processability of BDM but can also still maintain its great thermal stability. The reaction between $o, o^{\prime}$-diallylbisphenol $\mathrm{A}$ and BDM was suggested as through the "ene" reaction and Diels-Alder reaction between the ally groups and maleimide groups. ${ }^{7}$

Nevertheless, to further upgrade the BDM resins for composite application, two processing problems

* To whom correspondence should be addressed.

Journal of Applied Polymer Science, Vol. 51, 513-520 (1994)

(C) 1994 John Wiley \& Sons, Inc.

CCC 0021-8995/94/030513-08 remain to be overcome. One is that BDM is a crystalline solid with a melting point close to the initial cure temperature of the BDM-o,o'-diallylbisphenol A mixture. ${ }^{8}$ It often incurs partial curing during the preparation of a hot melt resin system. The other is that $\mathrm{BDM}$ tends to recrystallize from the mixture during ageing. ${ }^{9}$ As a result, the hot melt-type BDM$o, o^{\prime}$-diallylbisphenol A mixture often showed inconsistent properties.

Allylamine contains both an amine group and an allyl group and can react with BMI through the Michael addition and the reaction between allyl groups and benzene rings as well. ${ }^{10} \mathrm{~A}$ series of allylamine-adducted BMI (A-BMI), such as allylamine-adducted BDM (A-BDM) and allylamineadducted 4,4'-bismaleimidodiphenylether (A-BDE), were prepared by reacting BMI with various molar ratios of allylamine to reduce the crystallinity of $\mathrm{BMI}$, to improve the toughness of its cured resin, and to maintain its great thermal stability. In the first part of this study, ${ }^{10}$ two types of curing reactions for the A-BMI resins were found: the homopolymerization of A-BMI resins through opening of the residual double bonds in maleimide groups and the reactions between allyl groups and benzene rings 
in the BMI portion. In the second part of the study, we found that the domains produced from different types of curing reactions were immiscible. Therefore, the physical/mechanical properties of cured A-BMI resins were investigated in terms of their inhomogeneous morphology.

\section{EXPERIMENTAL}

\section{Sample Preparation}

The A-BMI samples, such as A-BDM and A-BDE prepared by reacting $B M I$ with various molar ratios of allylamine, are listed in Table I. A detailed description of the preparation method was given in the first part of the study. ${ }^{10}$ Curing of the A-BMI samples was conducted in a hot press by the following cure cycle: $2 \mathrm{~h}$ at $180^{\circ} \mathrm{C}$ plus $5 \mathrm{~h}$ at $220^{\circ} \mathrm{C}$ under $1.38 \times 10^{7} \mathrm{~Pa}$ pressure.

\section{Analytical Techniques}

The flexural properties of cured A-BMI were measured by the three-point bending test according to ASTM D-790. The support span for the three-point bending test is $4 \mathrm{~cm}$. Tests were performed in an Instron TM tensile tester at a crosshead speed of $0.5 \mathrm{~cm} \mathrm{~min}^{-1}$.

Dynamic mechanical analysis (DMA) of cured A-BMI was performed in a DuPont model 983 DMA. Tests were run at a heating rate of $10^{\circ} \mathrm{C} \mathrm{min}{ }^{-1}$.

The surface morphology of cured A-BMI was investigated by scanning electron microscopy (SEM). The fresh surfaces were made by fracturing the specimens immediately after they were quenched in liquid nitrogen. The fractured surfaces were gold-

Table I A-BMI Samples Prepared by Reacting BMI With Various Molar Ratios of Allylamine

\begin{tabular}{ccc}
\hline & \multicolumn{2}{c}{ A-BMI } \\
\cline { 2 - 3 } $\begin{array}{c}\text { Allylamine } \\
(\text { mol\%) }\end{array}$ & \% A-BDM & \% A-BDE \\
\hline 10 & 10 & 10 \\
50 & 50 & 50 \\
100 & 100 & 100 \\
150 & 150 & 150 \\
200 & 200 & 200 \\
\hline
\end{tabular}

a The molar percentage is calculated by dividing the moles of allylamine by the moles of BMI. sputtered and investigated in a JEOL JWM-5200 model SEM.

Thermogravimetric analysis (TGA) of the BMI and A-BMI was conducted in a Perkin Elmer TGA2 model thermogravimetric analyzer by heating from 50 to $800^{\circ} \mathrm{C}$ at a heating rate of $10^{\circ} \mathrm{C} \mathrm{min}{ }^{-1}$.

\section{RESULTS AND DISCUSSION}

\section{Dynamic Mechanical Properties and Morphology}

Dynamic mechanical analysis of the cured A-BMI was studied first. A broad glass-transition temperature $\left(T_{g}\right)$ peak at $186^{\circ} \mathrm{C}$ was found in the loss modulus curve of cured 10\%A-BDM [Figure 1(a)]. In addition, a $\beta$ relaxation peak was found at $-62^{\circ} \mathrm{C}$. As the amount of allylamine was increased to 50 mol \% for preparing the $50 \% \mathrm{~A}-\mathrm{BDM}$, the $T_{g}$ peak was shifted from 186 to $330^{\circ} \mathrm{C}$ as shown in Figure 1 (b). The intensity of the $\beta$ relaxation peak was substantially increased and a small additional peak appeared at $127^{\circ} \mathrm{C}$.

For the $10 \% \mathrm{~A}-\mathrm{BDM}$ cured mainly by homopolymerization through the opening of double bonds in maleimide groups, ${ }^{10}$ the broad $T_{g}$ peak should be contributed by the $\alpha$ relaxation of the homopolymerized network. Because of the fact that the amount of double bonds in 50\% A-BDM is less than in $10 \% \mathrm{~A}-\mathrm{BDM}$, the molecular weight of the homopolymerized network in the former resin would be smaller than in the latter, indicating that the small additional relaxation peak found at $127^{\circ} \mathrm{C}$ is contributed mainly by the homopolymerized network. In that case, because only two types of reactions, homopolymerization and the reactions between allyl groups and benzene rings, were found during cure of the A-BMI, ${ }^{10}$ the $T_{g}$ peak at $330^{\circ} \mathrm{C}$ should be associated with the network produced by the latter reaction.

The DMA results of cured A-BDE shown in Figure $2(a, b)$ are, in general, similar to those of cured A-BDM. However, the $T_{g}$ of cured $50 \% \mathrm{~A}-\mathrm{BDE}$ is $340^{\circ} \mathrm{C}$, higher than that of the cured $50 \% \mathrm{~A}-\mathrm{BDM}$. The intensity of the additional peak contributed by the homopolymerized network in the cured $50 \% \mathrm{~A}$ $\mathrm{BDE}$ is also higher than its counterpart in the cured $50 \%$ A-BDM, implying that the former resin has higher content of the domains contributed by the homopolymerized network than the latter. Thus, based on the DMA results, both cured 50\%A-BDE and $50 \% \mathrm{~A}-\mathrm{BDM}$ should have the inhomogeneous structure where the homopolymerized network and the network produced by the reaction between allyl groups and benzene rings are immiscible. To provide 


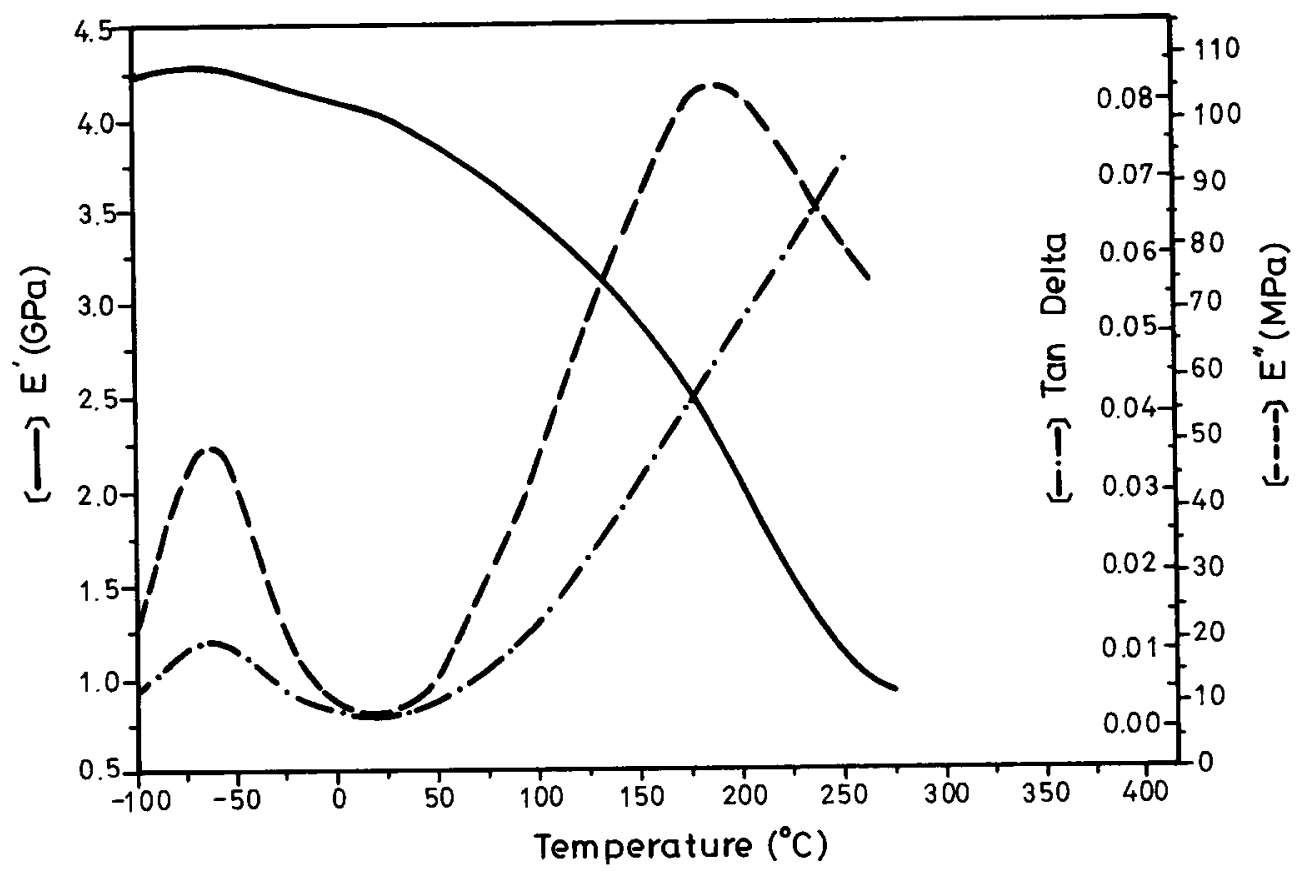

(a)

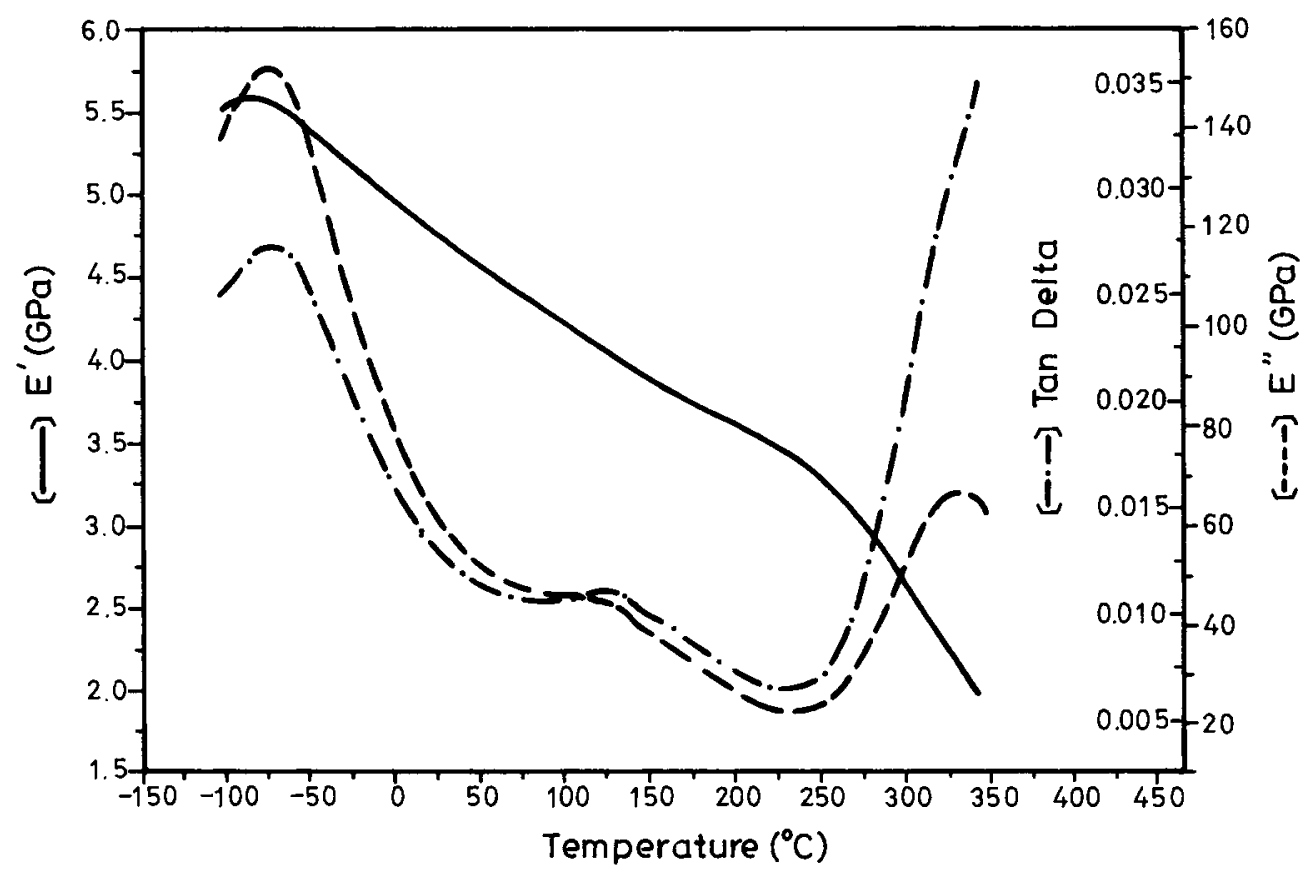

(b)

Figure 1 Dynamic mechanical spectra of the cured (a) $10 \%$ A-BDM and (b) $50 \%$ ABDM.

the direct evidence for the inhomogeneous structure, we employed SEM to investigate their fracture surface.

The fracture surface of both cured 10\%A-BDM and $10 \% \mathrm{~A}-\mathrm{BDE}$ shows a homogeneous structure as seen in Figures 3(a) and 4(a) which is consistent with the DMA results. As the amount of allylamine was increased to $50 \mathrm{~mol} \%$, the fracture surface of 


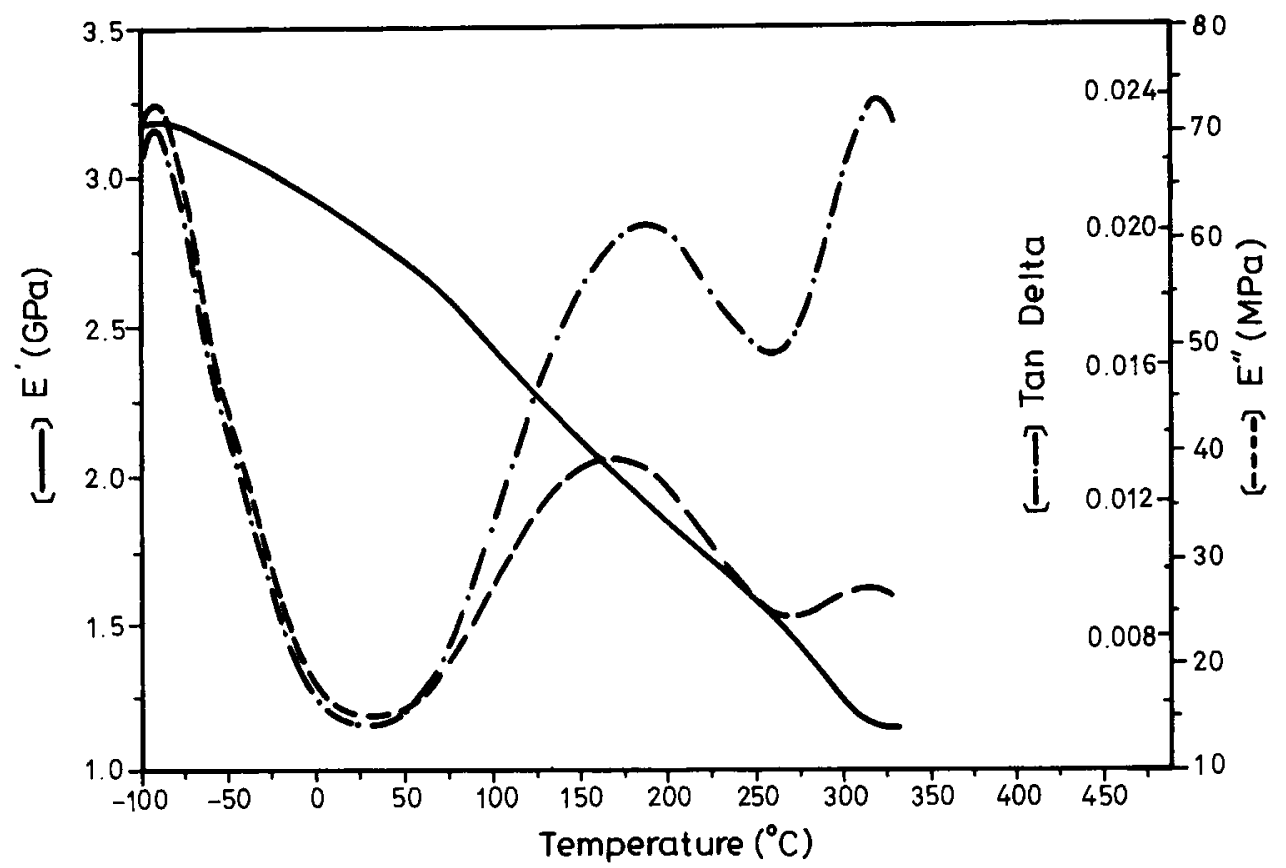

(a)

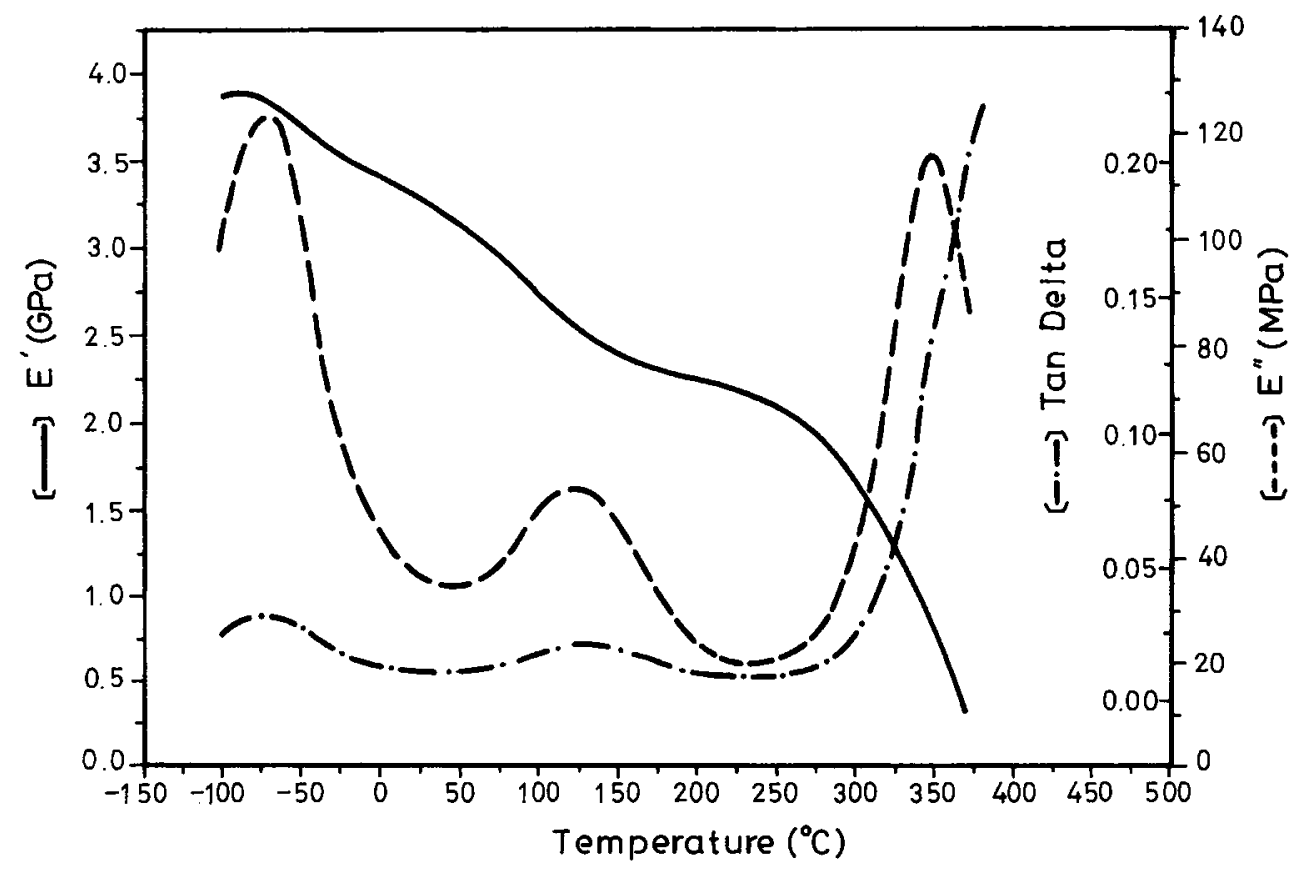

(b)

Figure 2 Dynamic mechanical spectra of the cured (a) 10\%A-BDE and (b) 50\%A-BDE.

cured $50 \% \mathrm{~A}-\mathrm{BDM}$ shows only roughness instead of two-phase morphology as seen in Figure $3(\mathrm{~b})$, whereas the fracture surface of cured $50 \% \mathrm{~A}-\mathrm{BDE}$ shows a clear two-phase morphology with a dispersed phase in a size range of 1-40 $\mu \mathrm{m}$ as seen in Figure 4(b). Two-phase morphology found in the cured $50 \% \mathrm{~A}-\mathrm{BDE}$ instead of the cured 50\%A-BDM might be due to the fact that the molecular chains containing ether groups in the former resin are more flexible than those containing $-\mathrm{CH}_{2}-$ groups in the latter, leading to the higher mobility of the molecular chains for phase separation. 


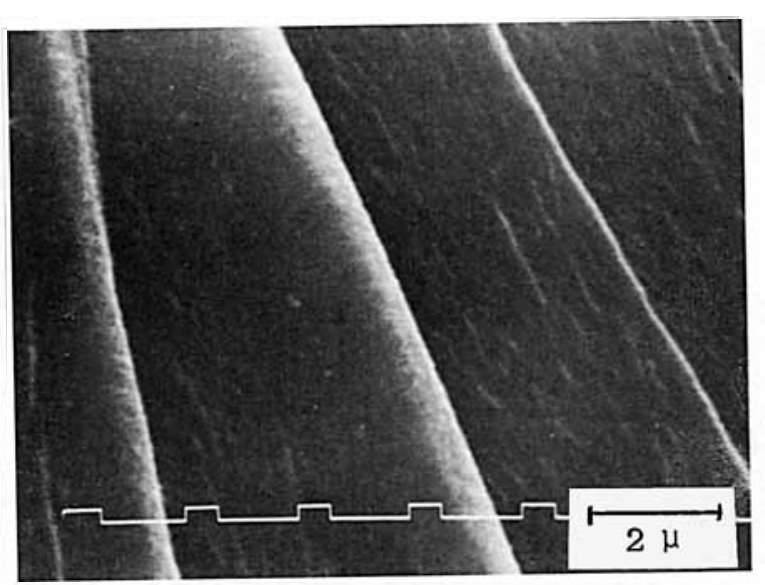

(a)

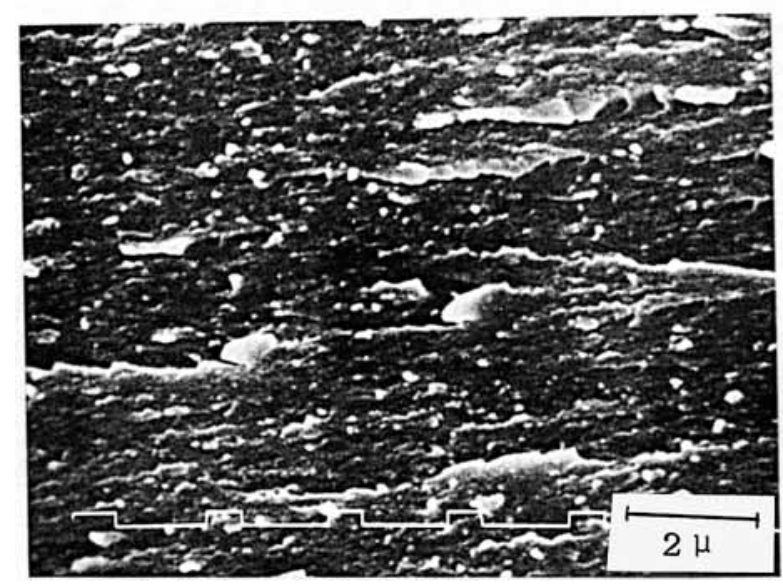

(b)

Figure 3 SEM photographs of the cured (a) $10 \% \mathrm{~A}$ BDM and (b) 50\%A-BDM.

Figure 5 shows the $T_{g}$ of cured A-BDM and ABDE measured from the loss modulus curve as a function of the molar ratio of allylamine used for monomer preparation. In general, the trend of change in $T_{g}$ is similar between the cured A-BDM and A-BDE. It reached the maximum as the molar ratio of allylamine reached $50 \mathrm{~mol} \%$ and then the $T_{g}$ decreased. The initial increase in $T_{g}$ came from the higher $T_{g}$ of the network produced by the reaction between allyl groups and benzene rings compared to the homopolymerized network. As the molar ratio of allylamine was over $50 \mathrm{~mol} \%$, the decrease in $T_{g}$ might be due to the fact that more aliphatic chain segments are introduced into the cured network.
In addition, the $\beta$ relaxation peak appeared at -100 to $-50^{\circ} \mathrm{C}$ range as shown in Figures $1(\mathrm{a}, \mathrm{b})$ and $2(\mathrm{a}, \mathrm{b})$, is associated with the local molecular motions in the glassy state. ${ }^{11}$ However, the exact type of molecular motion is not clear. The intensity of the $\beta$ relaxation peak in the loss modulus curve of cured A-BDM and A-BDE as a function of the molar ratio of allylamine used to prepare their monomers is shown in Figure 6. The highest intensity of $\beta$ peak was found in the cured $50 \% \mathrm{~A}-\mathrm{BDM}$ and $50 \% \mathrm{~A}-\mathrm{BDE}$ specimens, respectively. It was indicated that nearly all glassy polymers with high impact strength have prominent $\beta$ relaxation, ${ }^{12}$ sug-

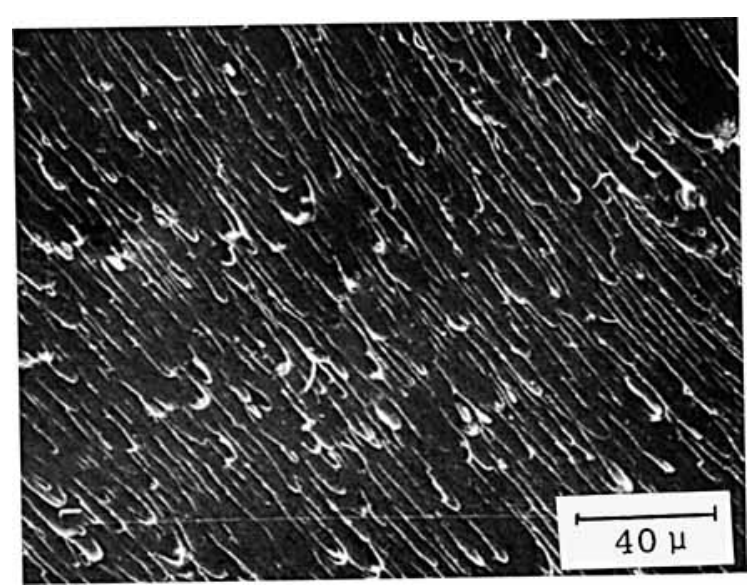

(a)

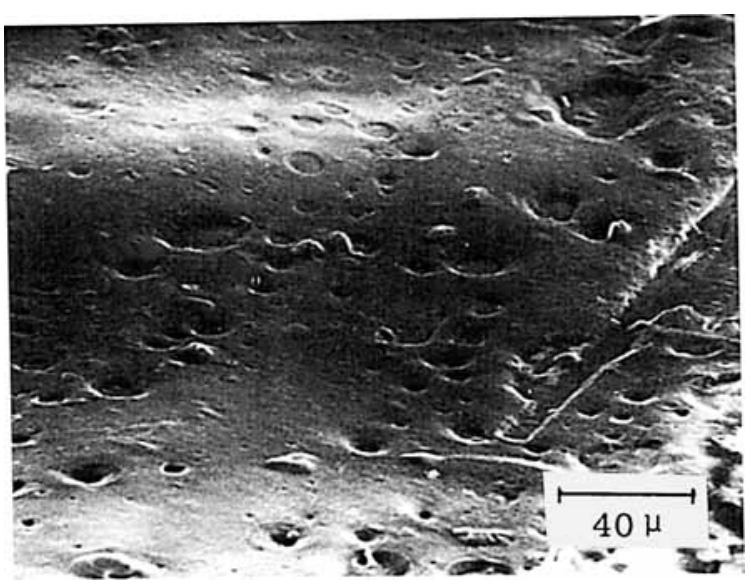

(b)

Figure 4 SEM photographs of the cured (a) 10\%A-BDE and (b) 50\%A-BDE. 


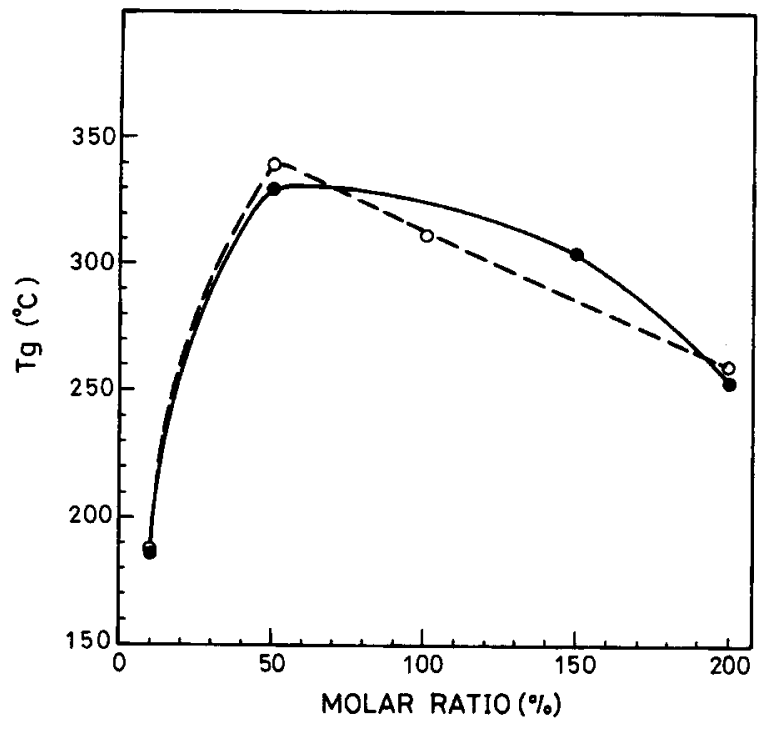

Figure $5 T_{g}$ of the cured (e) A-BDM and (O) A-BDE as a function of the molar ratio of allylamine used for monomer preparation.

gesting that the toughness of cured 50\%A-BDM and $50 \% \mathrm{~A}-\mathrm{BDE}$ might be the highest among all the cured $\mathrm{A}-\mathrm{BDM}$ and A-BDE samples. The detailed discussion on the mechanical properties of cured A-BMI is provided in the following section.

\section{Mechanical Properties and Decomposition Temperature}

The change in flexural strength of the cured A-BDM and $\mathrm{A}-\mathrm{BDE}$ as a function of molar ratio of allylamine used to prepare the monomers shown in Figure 7, is almost parallel to the change in the intensity of $\beta$ peak as seen in Figure 6. The unmodified BDM and BDE after cure were too brittle to measure the flexural strength. When they were modified by reacting with $10 \mathrm{~mol} \%$ allylamine, although the flexural strength of cured $10 \% \mathrm{~A}-\mathrm{BDM}$ and $10 \% \mathrm{~A}-\mathrm{BDE}$ could be measured, they were still brittle. Presumably, the cross-linking density provided by homopolymerization through opening of the double bonds is rather high. A significant increase in flexural strength was found as $50 \mathrm{~mol} \%$ allylamine was used to modify the BDM and BDE. It might be due to the fact that large amounts of the double bonds have been opened by allylamine so that the cross-linking density provided by homopolymerization is reduced. Besides, the flexural strength of cured 50\%A-BDE is higher than that of the cured 50\%A-BDM. When the amount of allylamine used was over $50 \mathrm{~mol} \%$, the flexural strength of both cured A-BDE and A-BDM

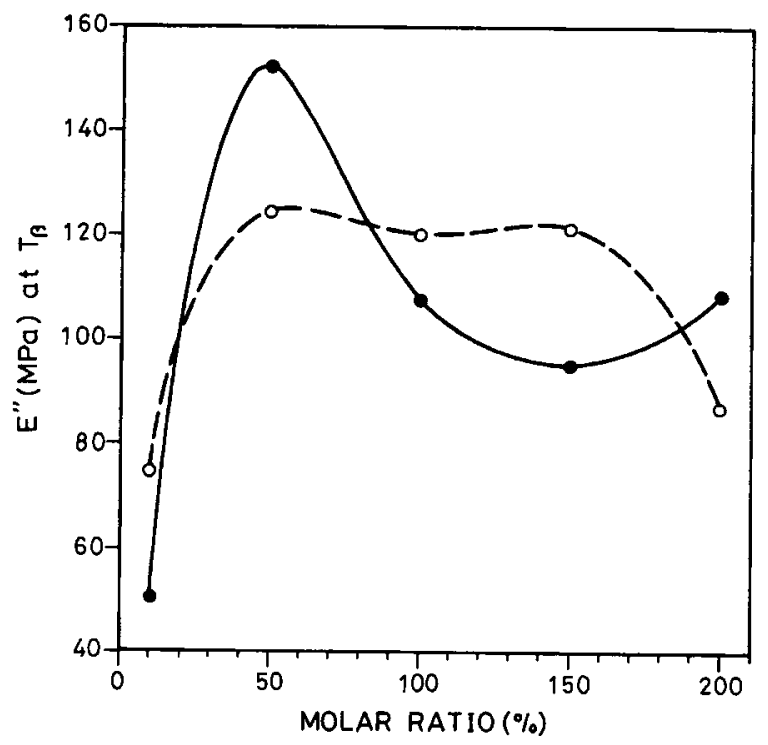

Figure 6 Intensity of $\beta$ relaxation peak of the cured (O) A-BDM and (O) A-BDE as a function of the molar ratio of allylamine used for monomer preparation.

was decreased and their morphology turned to a single phase mainly contributed by the reaction between allyl groups and benzene rings. Thus, the highest flexural strength of cured 50\%A-BDM and $50 \% \mathrm{~A}-\mathrm{BDE}$ among the cured A-BDM and A-BDE resins, respectively, might be attributed to their twophase morphology.

Furthermore, although the flexural strength of

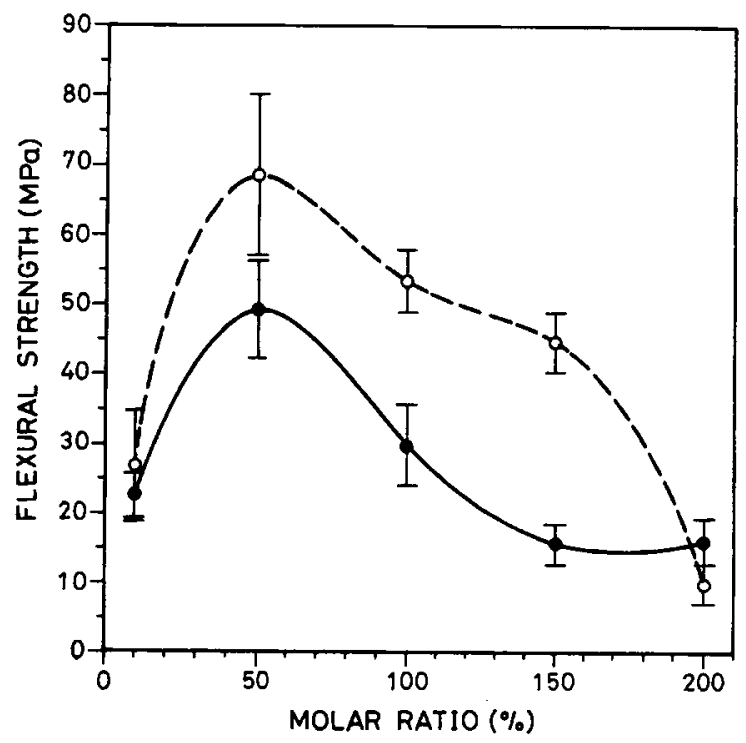

Figure 7 Flexural strength of the cured ( $)$ A-BDM and (O) A-BDE as a function of the molar ratio of allylamine used for monomer preparation. 
cured A-BDM and A-BDE is roughly parallel to the change in the intensity of $\beta$ peak (Figs. 6,7 ), the correlation between the flexural strength and the intensity of the $\beta$ peak is not clear. However, it was indicated that some type of $\beta$ relaxation appears to decrease notch sensitivity so that the polymer is able to sustain tensile loads. ${ }^{12}$ It was also reported that as the intensity of the $\beta$ peak is increased for the impact-modified polypropylene, its drop weight impact value is also increased, suggesting that a suitable calibration curve can be developed to predict the toughness by the intensity of the $\beta$ peak. ${ }^{13}$

The change in flexural modulus of cured A-BDM and A-BDE as a function of molar ratio of allylamine used to prepare the monomers is shown in Figure 8. For the cured A-BDM, the highest flexural modulus was found in the cured 50\%A-BDM. However, for the A-BDE, the highest flexural modulus was found in the cured $10 \% \mathrm{~A}-\mathrm{BDE}$. The modulus of polymeric materials in the rubbery and leathery states is directly related to the cross-linking density. However, in the glassy state, the resins are frozen to glass so that the cooperative motions of chain segments are prohibited. ${ }^{14}$ Consequently, the modulus is measured in the elastic region where the Van Der Waals attraction among the chain segments was affected. Thus, the modulus is not directly related to the cross-linking density but dependent on the packing density or concentration of the chain segments in the glassy state. For the cured A-BDM, the maxi-

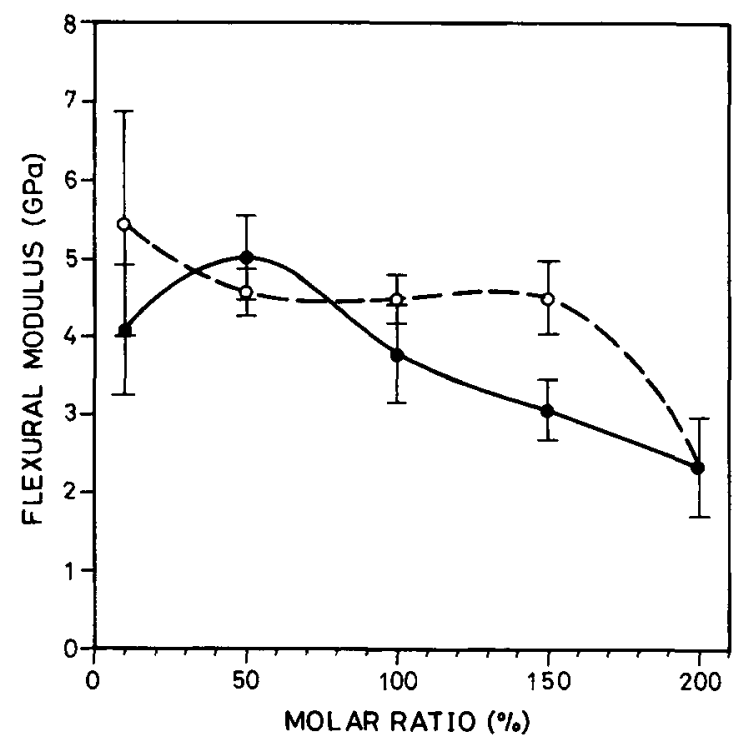

Figure 8 Flexural modulus of the cured (๑) A-BDM and $(O)$ A-BDE as a function of the molar ratio of allylamine used for monomer preparation.

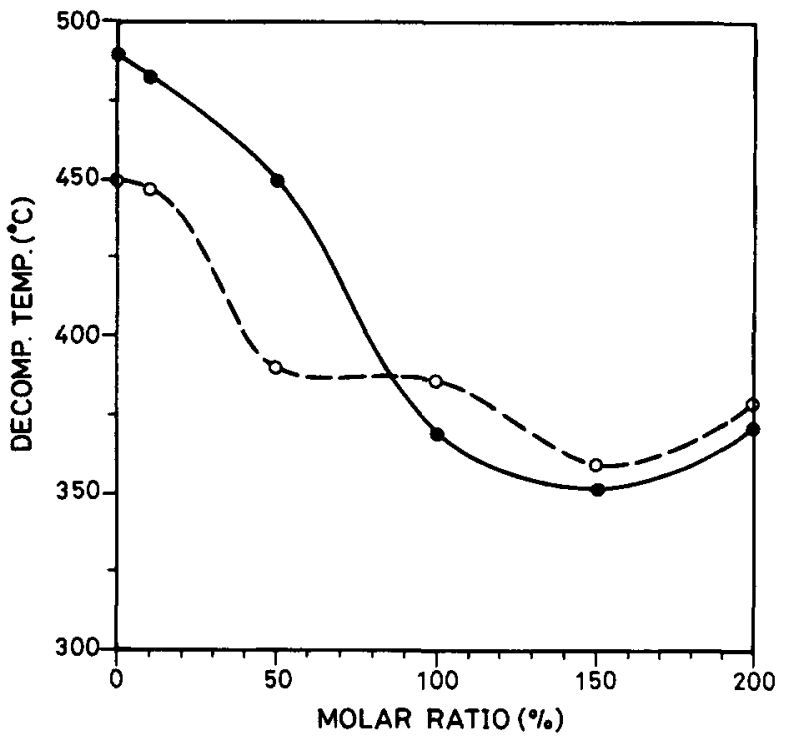

Figure 9 Degradation temperature of ( ) A-BDM and (O) A-BDE as a function of the molar ratio of allylamine to react with $B D M$ and $B D E$, respectively.

mum flexural modulus found in the cured $50 \% \mathrm{~A}$ BDM might be due to the fact that the network produced by homopolymerization and that produced by the reactions between allyl groups and benzene rings are more compatible and might interpenetrate with each other, resulting in the increase in packing density of the molecular segments in view of less phase separation compared to the cured 50\%A-BDE.

The degradation temperature $\left(T_{d}\right)$ of BDM, A$\mathrm{BDM}, \mathrm{BDE}$, and A-BDE at the $5 \mathrm{wt} \%$ loss of materials measured by the thermogravimetric analysis is shown in Figure 9. The $T_{d}$ of pure BDM is $490^{\circ} \mathrm{C}$ with $50 \mathrm{wt} \%$ char yield at $800^{\circ} \mathrm{C}$. When it was modified by $50 \mathrm{~mol} \%$ allylamine, the $T_{d}$ only dropped to $450^{\circ} \mathrm{C}$. But with a further increase of the amount of allylamine to $100 \mathrm{~mol} \%$, the $T_{d}$ dropped to $369^{\circ} \mathrm{C}$ with $48 \mathrm{wt} \%$ char yield. On the other hand, the $T_{d}$ of pure $\mathrm{BDE}$ is $450^{\circ} \mathrm{C}$ with the same char yield as $\mathrm{BDM}$ at $800^{\circ} \mathrm{C}$. When it was modified by $50 \mathrm{~mol} \%$ allylamine, the $T_{d}$ dropped at $390^{\circ} \mathrm{C}$ with the char yield maintained at $50 \mathrm{wt} \%$. With a further increase in the amount of allylamine, the $T_{d}$ was not significantly changed. In summary, the $T_{d}$ of A-BDM was substantially decreased as the amount of allylamine was increased from 50 to $100 \mathrm{~mol} \%$ and then leveled off; whereas the substantial decrease in $T_{d}$ of the ABDE took place earlier. However, the char yield for both A-BDM and A-BDE was only slightly changed by increasing the amount of allylamine.

Finally, according to the experimental results, the 
cured 50\%A-BDM not only has the highest $T_{g}$ and the highest flexural strength and modulus among the cured A-BDM, but it still has high degradation temperature. In addition, its processability is better than that of the unmodified BDM in view of the low crystallinity and melting point. ${ }^{10}$ Thus, its application to the composite materials as a resin matrix is promising and will be studied in the near future.

\section{CONCLUSIONS}

During cure of the A-BDM and A-BDE, the network produced by homopolymerization through the opening of double bonds in maleimide groups is immiscible with that produced by the reactions between the allyl groups and benzene rings. Due to the fact that the former network has much higher $T_{g}$ than the latter, the $T_{g}$ of cured BDM and BDE was significantly increased by the presence of the network produced by the latter reactions. Among the cured A-BDM, the cured 50\%A-BDM has the optimal physical/mechanical properties, such as the highest $T_{g}$, the highest intensity of $\beta$ relaxation, the highest flexural strength and modulus, and still has high degradation temperature. On the other hand, although the $T_{g}$, the intensity of $\beta$ relaxation, and flexural strength of cured 50\%A-BDE are the highest among the cured A-BDE, its degradation temperature is rather low.

Financial support of this work by the National Science Council in Taiwan, R.O.C., through Grant NSC81-0405E002-09 is gratefully acknowledged.

\section{REFERENCES}

1. J. V. Crivello, J. Polym. Sci., Polym. Chem. Ed., 11, 1185 (1973).

2. I. K. Varma, G. M. Fohlen, and J. A. Parker, J. Polym. Chem. Ed., 20, 283 (1982).

3. D. K. Kumar, G. M. Fohlen, and J. A. Parker, J. Polym. Sci., Polym. Chem. Ed., 21, 245 (1983).

4. I. K. Varma, Sangita, and D. S. Varma, J. Polym. Sci., Polym. Chem. Ed., 22, 1419 ( 1984 ).

5. C. D. Giulio, M. Gautier, and B. Jasse, J. Appl. Polym. Sci., 29, 1771 (1984).

6. J. King, M. Chaudhari, and S. Zahir, Proceedings of the 29th National SAMPE Conference, 29, 392 (1984).

7. M. Chaudhari, T. Galvin, and J. King, SAMPE J., 21, 17 (1985).

8. J. Mijovic and B. Schafran, SAMPE J., 26, 51 (1990).

9. H. D. Stenzerberger, W. Roemer, M. Herzog, S. Pierce, M. Canning, and K. Fear, 31st Intl. SAMPE Symp., 920 (1986).

10. K.-F. Lin and J.-S. Lin, to appear.

11. J. D. Ferry, Viscoelastic Properties of Polymers, 3rd ed., John Wiley, New York, 1980, p. 437.

12. L. E. Nielsen, Mechanical Properties of Polymers and Composites, Vol. 1, Marcel Dekker, New York, 1974, p. 215.

13. R. Hassel, private communication.

14. P. Meares, Polymers: Structure and Bulk Properties, Van Nostrand, Princeton, New Jersey, 1965, p. 251.

Received December 21, 1992

Accepted July 1, 1993 